\title{
Sensorimotor beta power reflects the precision-weighting afforded to sensory prediction errors
}

Clare E Palmer1,2, Ryszard Auksztulewicz³ ${ }^{3}$ Sasha Ondobaka1 \& James M Kilner ${ }^{*}$

${ }^{1}$ Department of Clinical and Movement Neurosciences, UCL, London UK, WC1N 3BG

${ }^{2}$ Center for Human Development, University of California San Diego, La Jolla, CA 92093

${ }^{3}$ Department of Biomedical Sciences, City University of Hong Kong, Hong Kong SAR

*Corresponding author: j.kilner@ucl.ac.uk

No conflicts of interest.

\section{Acknowledgements}

This research was conducted as part of CEP's PhD work funded by the 4-year Wellcome Trust PhD studentship in Neuroscience at UCL. 


\section{ABSTRACT}

It has been proposed that accurate motor control relies on Bayesian inference that integrates sensory input with prior contextual knowledge (Bays \& Wolpert, 2007; Körding \& Wolpert, 2004; Wolpert, Ghahramani, \& Jordan, 1995). Recent evidence has suggested that modulations in beta power $(\sim 12-30 \mathrm{~Hz})$ measured over sensorimotor cortices using electroencephalography (EEG) may represent parameters of Bayesian inference. While the well characterised postmovement beta synchronisation has been shown to correlate with prediction error (H. Tan, Jenkinson, \& Brown, 2014; Huiling Tan, Wade, \& Brown, 2016), recent evidence suggests that beta power may also represent uncertainty measures (Tan et al., 2016; Tzagarakis, West, \& Pellizzer, 2015). The current study aimed to measure the neurophysiological correlates of uncertainty mediating Bayesian updating during a visuomotor adaptation paradigm in healthy human participants. In particular, sensory uncertainty was directly modulated to measure its effect on sensorimotor beta power. Participant's behaviour was modelled using the Hierarchical Gaussian Filter (HGF) in order to extract the latent variables involved in learning actions required by the task and correlate these with the measured EEG. We found that sensorimotor beta power correlated with inverse uncertainty afforded to sensory prediction errors both prior to and following a movement. This suggests that sensorimotor beta oscillations may more readily represent relative uncertainty within the sensorimotor system rather than error. Neurophysiological models describing the generation of beta oscillations offer a potential mechanism by which this neural signature may encode latent uncertainty parameters. This is essential for understanding how the brain controls behaviour.

\section{HIGHLIGHTS}

- The functional role of sensorimotor beta oscillations is unknown, however increasing evidence suggests that beta power is associated with Bayesian inference important for motor control.

- This study furthers these findings by highlighting that beta power both prior to and following a movement readily correlates with the precision-weighting afforded to sensory prediction errors.

- Isolating prediction error signalling from its precision-weighting is important for understanding how Bayesian inference may occur in the brain and the underlying function of beta oscillations in the sensorimotor system.

- A unified theory of sensorimotor beta activity may account for behavioural impairments in patients with movement disorders in which beta power is abnormal, such as Parkinson's Disease.

- Developing novel hypotheses for how a well-characterised neurophysiological impairment may contribute to behavioural symptoms may prompt the development of novel therapies.

\section{INTRODUCTION}

Accurate motor control under uncertainty has been proposed to rely on Bayesian inference, which integrates incoming sensorimotor information with prior contextual knowledge (Bays \& Wolpert, 2007; Körding \& Wolpert, 2004; Wolpert et al., 1995). The sensorimotor system is thought to represent a generative model, used to predict the sensory consequences of a 
movement (Bastian, 2006; Blakemore \& Sirigu, 2003; Wolpert \& Miall, 1996). Predictions of the generative model are compared with cortical reafference to produce prediction errors (PEs), which in turn update the internal model to generate more accurate predictions in the future to guide behaviour. This hierarchical message passing has been hypothesised to occur via the coupling of oscillatory activity across cortical layers and between distant cortical regions (Arnal \& Giraud, 2012; Arnal, Wyart, \& Giraud, 2011; Bastos et al., 2012). Indeed, within the sensorimotor system, beta oscillations $(\sim 12-30 \mathrm{~Hz})$, which robustly desynchronise with movement and resynchronise following movement (Davis, Tomlinson, \& Morgan, 2012; Engel \& Fries, 2010; Simon Little \& Brown, 2014), have been hypothesised to have a functional role in Bayesian inference important for motor control. The post-movement beta synchronisation (PMBS) has been shown to correlate with the magnitude of PE following an unexpected perturbation to a movement trajectory (H. Tan, Jenkinson, et al., 2014; Huiling Tan et al., 2016) and has been associated with the update of the motor command needed to correct for previous errors in a visuomotor adaptation paradigm (Torrecillos, Alayrangues, Kilavik, \& Malfait, 2015).

Importantly, estimates of uncertainty determine how readily PEs update the internal model. For example on a foggy day, uncertain visual information will produce less precise (more uncertain) PE signals, such that the individual will rely more on prior beliefs to generate predictions that guide behaviour rather than the visual information. However, previous studies highlighting the relationship between beta power and PE have not readily dissociated between the magnitude of the error signal and its precision weighting. This is important as evidence suggests that sensorimotor beta power may reflect uncertainty estimates associated with the motor prediction (Tan et al., 2016; Tzagarakis, West, \& Pellizzer, 2015). We have previously hypothesised that beta power may correlate with uncertainty in the afferent input used to generate PEs (Palmer, Zapparoli, \& Kilner, 2016). This is supported by circumstantial evidence that the time course of beta desynchronisation accompanying movement correlates with somatosensory attenuation, which is thought to reflect a reduction in the gain of incoming afferent information to the cortex (Cohen \& Starr, 1987; Davis et al., 2012; Engel \& Fries, 2010; Starr \& Cohen, 1985). Indeed, it is hypothesised that precision (inverse uncertainty) is encoded by synaptic gain (post-synaptic responsiveness) on superficial pyramidal cells (K. Friston \& Kiebel, 2009; K. Friston, Mattout, \& Kilner, 2011). Neuronal oscillations have been shown to both modulate synaptic gain and be generated by changes in synaptic gain, therefore this model provides a neurophysiological mechanism by which beta oscillations could either be the cause or consequence of modulations in precision.

In the current study, we aimed to measure the neurophysiological correlates of states mediating Bayesian updating during a visuomotor adaptation paradigm. We induced PEs behaviourally, by adding an angular rotation into the visual feedback of participants' movements and specifically manipulated sensory precision by adding visual noise to the feedback. In this way, we orthogonalised PE and sensory precision by design. We also modelled participant's behaviour using the Hierarchical Gaussian Filter (HGF) in order to extract the latent variables involved in learning actions required by the task (C. D. Mathys et al., 2014; C. Mathys, Daunizeau, Friston, \& Stephan, 2011). We posit that sensorimotor beta power may reflect uncertainty in the sensorimotor system. However, there are multiple components of beta power modulation with movement, therefore different uncertainty estimates may dominate the signal at different time points. Indeed, a recent study has shown that beta power prior to and following a movement localises to different neural substrates (Alayrangues, Torrecillos, Jahani, 
\& Malfait, 2019). We therefore hypothesised that: 1) sensorimotor beta power prior to movement would correlate with prior precision associated with sensorimotor predictions; 2) sensorimotor beta power following a movement would correlate with sensory precision associated with sensory prediction errors.

\section{METHODS}

\section{Participants}

24 healthy participants (female $=12$ ) aged 21-37 years old (mean \pm SD: $25.46 \pm 4.56$ ) took part in this study. Participants had no history of neurological or psychiatric illness by self-report. All participants were right handed and gave written informed consent prior to taking part. This study was approved by the UCL Research Ethics Committee and all testing took place at the UCL Institute of Neurology, Queen Square. 5 participants were excluded for showing no visuomotor adaptation in the behavioural task (no significant difference in angular error between the angular perturbation switch (APS) trial and the following tenth trial, $\mathrm{p}>0.1$; see Behavioural Data Analysis for more details). This suggested they were not following the task instructions correctly. All subsequent analyses were carried out on 19 participants (female=9) aged 21-34 years old (mean \pm SD: $25.53 \pm 4.03$ ).

\section{Experimental Setup}

Participants were seated in front of a laptop with their dominant hand resting on a trackpad (Sway MultiTouch Trackpad, Speedlink, London) under a box, which hid their arm from view. Participants completed the task by sliding their finger in 2D along the trackpad, which was positioned perpendicular to the monitor providing visual feedback of the movement. The position of the tip of their finger on the trackpad was shown as a circular cursor on the screen during the task. The sampling rate was capped at the $60 \mathrm{~Hz}$ refresh rate of the monitor. Participants completed a visuomotor adaptation task (custom code using Cogent 2000 in Matlab 2013b). EEG data were recorded using a BioSemi 128 active electrode system at a sampling frequency of $2048 \mathrm{~Hz}$. Two external reference electrodes were placed on the participants' earlobes.

\section{Task Procedure: Visuomotor Adaptation Task}

The task design can be seen in Figure 1 and was adapted from similar tasks used previously (H. Tan, Jenkinson, et al., 2014; H. Tan, Zavala, et al., 2014; Huiling Tan et al., 2016; Torrecillos et al., 2015). A start position (red circle) was presented at the bottom of a black screen at the start of a trial for $4 \mathrm{~s}$. The start position turned orange indicating a READY signal and remained on screen for $1.5 \mathrm{~s}$. At the GO signal, the start position disappeared and a single target appeared $20 \mathrm{~cm}$ above the start position. The target consisted of a small filled in circle at the centre of a larger outer circle. A circular cursor appeared on screen at the position of the tip of the participants' index finger on the trackpad. Participants were instructed to move the cursor into the target zone following the most direct path as quickly and accurately as possible. This was to encourage participants to explicitly adapt to the angular error. A trial ended when participants reached the target zone. On reaching the target participants were instructed to remain still for $2 \mathrm{~s}$ and then when given the RETURN signal were instructed to return to the start position without visual feedback. A tactile marker on the track pad indicated to the participant that they 
were back in the start position. The ISI between the RETURN signal and the READY signal for the next trial was 4s. There were 387 trials in total with 43 trials per block and 9 blocks. The first block was a training block and was not included in subsequent analyses. Participants were given a few minutes rest between blocks.

The visual feedback of the cursor given during the trial was perturbed in two ways, which were independent from each other. Firstly, an angular rotation was introduced on certain trials such that the position of the cursor on the screen did not always directly emulate the position of the participant's finger on the trackpad. The angular rotation in the cursor was either 30 degrees, 60 degrees or was veridical with the participant's finger position (Figure 1B). When an angular rotation was introduced on an angular perturbation switch (APS) trial, this remained constant for 10 trials and then at a random trial number between 11 and 15 a new angular rotation was introduced. However, participants were told that a new angular rotation could be introduced at any point after 7 trials. The sequence of angular rotations was pseudorandomised but the number of different changes in angular rotation was controlled such that there were an equal number of small and large APS trials. Change in angular rotation was considered large if the rotation changed from $0^{\circ}$ to $60^{\circ}$ or vice versa and was considered small if the rotation changed from $0^{\circ}$ to $30^{\circ}$ or $30^{\circ}$ to $60^{\circ}$ and vice versa. Secondly, a displacement in the X-axis was added into the visual feedback of the cursor and varied along the cursor trajectory in "high visual noise" blocks (Figure 1C). This displacement was randomly sampled at each point of the movement trajectory from a normal distribution $(\mu=0 \mathrm{~cm}, \sigma=\sim 3 \mathrm{~cm})$. This made the cursor jitter along the $\mathrm{X}$-axis throughout the trial, which made it difficult to accurately determine the exact position of the cursor at any given point on a given trial and therefore provided a manipulation of the reliability of the visual feedback, which can be likened to precision. In "no visual noise" blocks the cursor accurately tracked the participant's finger position without any added displacement except for the angular rotation. The type of block alternated and the noise level of the first block was counterbalanced across participants, except for the training block, which was always a "no visual noise" block.

Each block started with the adapted angular rotation from the previous block and a new rotation was given after 3 trials. This was to ensure that the noise perturbation and an angular perturbation did not occur at the same time. Participants were explicitly told this and the first 3 trials of each block were excluded from data analysis.

\section{Behavioural Data Analysis}

Data were analysed using custom code written in MATLAB (version 2013b; MathWorks). There were four main behavioural dependent variables analysed in this task: angular error, average velocity, movement time and path length. Velocity was calculated from the differentiated cursor position and convolved with a Gaussian kernel with a full-width-half-maximum (FWHM) of $40 \mathrm{~ms}$. Velocity value across the whole movement trajectory were averaged to calculate average velocity (AV). Angular error (AE) was calculated as the angle between a line connecting the start position and the target and a line connecting the start position and the point of maximum velocity. Movement initiation was calculated as the point in which the cursor crossed a semicircular boundary with a radius of $5 \mathrm{~mm}$ from the start position. Movement offset was defined as the time at which the cursor reached the outer circle of the target zone. Movement time (MT) was calculated as the difference between the time of movement offset and the time of 
movement initiation. Path length (PL) was calculated as the sum of the differentiated cursor position from the time of movement initiation to the time of movement offset. If a participant did not respond correctly on a given trial, that trial was excluded from all analyses based on the following criteria: 1) participants did not return to the start position in time on the previous trial demonstrated by movement during a $200 \mathrm{~ms}$ window following the onset of the ready signal; 2) movement initiation was quicker than $200 \mathrm{~ms}$ following the onset of the go signal; or, 3) participants did not move following the go signal. This resulted in a mean (sd) of 3.31 (1.56) trials excluded per subject across the whole task. A 2x2x10 repeated measures ANOVA with factors change in angular rotation, visual noise and repetition number was used to analyse each dependent variable. All comparisons were corrected for multiple comparisons using the Bonferroni method where applicable. Where assumptions of sphericity were not met the Greenhouse-Geisser (GG) correction was applied.

\section{Behavioural modelling using the Hierarchical Gaussian Filter (HGF)}

We aimed to determine the neurophysiological correlates of hidden beliefs modelled using a two-level HGF (Figure 2). In this model Bayesian updating occurs at multiple (here: two) levels in a hierarchy and the volatility, or uncertainty, at each level of the hierarchy is determined by the volatility of the hidden state at the level below. Hidden states evolve over time at each level via a Gaussian random walk with its variance (volatility or step size) coupled to the level above such that different levels of uncertainty are represented at different levels of the hierarchy. The HGF consists of two models. The perceptual model determines how beliefs evolve over time given specific task inputs. The response model then maps those beliefs onto actions by determining how a subject should behave given the estimated hidden states. In fitting the HGF to the data, participant's behavioural responses are used to estimate specific parameter values that best explain how the beliefs described by the perceptual model influence that individual's behaviour. For example, individual estimates of learning rate based on the behavioural data determine how quickly a participant will adapt to changes in perturbation level across trials. The output of the HGF is several time-series, which demonstrate the trial-wise evolution of hidden beliefs throughout the task that are individual to each subject. These trajectories were then correlated with neurophysiological data (induced EEG activity; see below) to understand how these beliefs were represented in the brain in this task.

\section{i) The Perceptual Model}

In this model beliefs about hidden states were updated over time using a mean-field approximation to Bayesian belief updating. Hierarchical levels were linked by the predictions of hidden states at lower levels, and the ensuing precision-weighted PEs updated the predictions at higher levels. Estimates of uncertainty and individual learning rates were used to weight PEs at different levels of the hierarchy to explain the behavioural data. In this task, the manipulation of uncertainty was twofold: (1) which visuomotor perturbation level would govern a given trial $(0,30$, or 60 degrees); and (2) how much noise there would be in the visual feedback. The angular perturbation level changed implicitly over the course of the experiment, while feedback noise was manipulated explicitly and presented in separate blocks. The model estimated the participants' trial-by-trial beliefs about the angular perturbation level, corresponding to the lowest level in the model, denoted by $x_{1}$. Participants were told that the perturbation would change on a random trial between 7-15 trials after it was introduced. Over the course of the experiment, participants could learn when the perturbation level was more likely to change; 
accordingly, the beliefs about the higher-level structure of the task are denoted by $\mathrm{x}_{2}$. The inferred beliefs constitute the hidden states of an observation model (C. Mathys et al., 2011) and evolve as a Gaussian random walk. The generative model is hierarchical, i.e., the hidden states at a given level determine the variance of the random walk at the level below:

(1) $p\left(x_{1} \mid x_{2}^{(k)}\right)=N\left(x_{1} ; x_{2}^{(k)}, \alpha\right)$,

(2) $p\left(x_{2}^{(k)} \mid x_{2}^{(k-1)}\right)=N\left(x_{2}^{(k)} ; x_{2}^{(k-1)}, \exp (\omega)\right)$.

At the lower level (Eq. 1), the prediction of the visuomotor rotation depended on the current task representation level and visual feedback noise $\alpha$ (assumed to be constant for a given block). At the higher level (Eq. 2), the inferred task representation level in a given trial $x_{2}^{(k)}$ was normally distributed around the validity level from the previous trial $x_{2}^{(k-1)}$, with the variance of this distribution depending on the learning step size $\omega$. In this paradigm the validity of the visuomotor perturbation was fixed at $100 \%$ (i.e., all trials governed by e.g. a $30^{\circ}$ rotation required a $30^{\circ}$ displacement of the motor action relative to the visual input to accurately hit the target), however the second level of the observation model can efficiently learn about probabilistic validity levels (cf. e.g.(Vossel et al., 2014)). This model can in principle be extended with further hierarchical levels (C. D. Mathys et al., 2014) describing e.g. the volatility of the perturbation level; however, in our paradigm no further manipulations of uncertainty were required.

During the fitting of the model to the data, one can estimate the trial-by-trial time-series (at each level i) of the participants' beliefs $\mu_{i}^{(k)}$ (i.e., posterior means of states $x_{i}^{(k)}$ ) and the updates on these beliefs $\varepsilon_{i}^{(k)}$ (precision-weighted PEs) after observing an outcome. The variational approximation in the HGF provides analytic update equations describing these time-series:

(3) $\mu_{2}^{(k+1)}-\mu_{2}^{(k)} \sim \psi_{2}^{(k)} \delta_{1}^{(k)}=\varepsilon_{2}^{(k)}$,

(4) $\psi_{2}^{(k)}=\frac{\widehat{\pi}_{1}^{(k)}}{\pi_{2}^{(k)}}$

(5) $\pi_{2}^{(k)}=\frac{1}{\sigma_{2}^{(k)}}$

(6) $\delta_{1}^{(k)}=\frac{\sigma_{1}^{(k)}+\left(\mu_{1}^{(k)}-\mu_{1}^{(k-1)}\right)^{2}}{\sigma_{1}^{(k-1)}}-1$.

As shown in equations 3-6, in each trial, a belief update at the second level, about the task representation $\mu_{2}^{(k+1)}-\mu_{2}^{(k)}$ is proportional to the PE at the level below $\delta_{1}^{(k)}$, weighted by a precision ratio $\psi_{2}^{(k)}$. This precision ratio depends on the precision (inverse variance) of the prediction at the level below, $\hat{\pi}_{1}^{(k)}$, and the precision at the current level $\pi_{2}^{(k)}$. The superscript ${ }^{\wedge}$ denotes the prediction before observing the trial outcome; accordingly, $\hat{\pi}_{i}^{(k)}$ is the precision of this prediction. At the lower level, the updates take a similar form. The numerator of the 
precision term includes the parameter of sensory uncertainty and therefore the precision weighting modulates according to the noise level of the visual feedback.

(7) $\mu_{1}^{(k+1)}-\mu_{1}^{(k)}=\psi_{1}^{(k)} \delta_{1}^{(k)}$,

(8) $\psi_{1}^{(k)}=\frac{1}{\alpha \pi_{1}^{(k)}}$

(9) $\hat{\pi}_{1}^{(k)}=\frac{1}{\sigma_{1}^{(k-1)}+e^{\omega}}$

$\delta_{u}^{(k)}=u^{(k)}-\hat{\mu}_{1}^{(k)}=u^{(k)}-\mu_{1}^{(k-1)}$

(11) $\pi_{1}^{(k)}=\hat{\pi}_{1}^{(k)}+\frac{1}{\alpha}$

$$
\pi_{2}^{(k)}=\pi_{2}^{(k-1)}+\frac{1}{\widehat{\pi}_{1}^{(k)}}
$$

At the lower level, the PE about the observed perturbation level $\delta_{1}^{(k)}$ is simply the difference between the actual and the predicted outcome, where the prediction is inherited from the previous trial (Eq. 10). This PE, weighted by its variance and sensory noise $\alpha$, is used to update the predictions about the outcome in the next trial (Eq. 7\&8). At the higher level, the PE about the visuomotor perturbation level is used to update the prediction of its validity in the next trial (Eq. 3). The precision (inverse variance) is updated according to equations 11 and 12 . These HGF-derived time-series - fitted to each participant's behavioural data - were used as regressors in subsequent analysis of EEG data. Initial variance parameters $\log \left(\sigma_{1,2}^{(0)}\right)$ were fitted to individual participant's data together with the remaining model parameters.

\section{ii) The Response Model}

To map the estimated hidden states (beliefs) onto the observed behavioural data, we specified a response model for the measured movement time (MT). Visuomotor adaptation studies have previously controlled for movement speed, such that the only variable to vary from trial to trial is angular error, and this is used as a measure of adaptation. However, the speed in which a participant moves contains a lot of important information related to how confident they are, which is thought to reflect estimates of precision. We therefore decided to use movement time as our dependent variable for the response model as this encompasses both speed and accuracy in a single variable and therefore produces a more holistic summary of how participants behaved. The response model was based on the trial-by-trial estimate of surprise, $S$ (Vossel et al., 2014):

$$
\begin{aligned}
& M T=\zeta_{0}+x_{1}\left(\zeta_{p}+\zeta_{2} S\right)+\left(1-x_{1}\right)\left(\zeta_{n}+\zeta_{2}(1-S)\right), \\
& S=\frac{1}{1+\operatorname{surprise}\left(\widehat{\mu}_{1}\right)}=\frac{1}{1-\log _{2}\left(\widehat{\mu}_{1}\right)} .
\end{aligned}
$$

Inputs $x_{1}$ were coded such that they mapped onto a range $\{0,1\}$ corresponding to $\left\{0^{\circ}, 60^{\circ}\right\}$ displacement with a $30^{\circ}$ displacement corresponding to $x_{1}=0.5$. Responses MT were calculated as response speed, i.e., the reciprocal of movement times. A time-resolved value $S$ represents attentional resources, scales with Shannon surprise associated with the target stimulus, and respects the same boundary conditions as responses and inputs, i.e., is confined to the $\{0,1\}$ 
interval with $S=0.5$ when $\hat{\mu}_{1}=0.5$. Parameters $\zeta$ quantify each participant's MT values $\left(\zeta_{0}\right.$ : baseline; $\zeta_{p}$ and $\zeta_{n}$ : MT contribution after increasing and decreasing the perturbation level respectively), with $\zeta_{2}$ denoting the weight of the attentional resources onto a given trial's estimated MT.

\section{Model comparison}

In order to determine that participants were behaving in a Bayes optimal manner throughout this task and therefore validate the use of the HGF in this study, we compared two observation models (HGF and a standard reinforcement learning model; cf. Rescorla and Wagner, (1972)) using Bayesian Model Selection (BMS). The Rescorla-Wagner (RW) learning model demonstrates how the association between a conditioned stimulus (CS) and an unconditioned stimulus (US) is learned over time. This association is updated in a trialwise manner depending on a prediction error weighted by a constant salience term that does not vary over time, but allows the learning rate to vary on an individual basis. In this experiment the CS can be likened to the angular rotation in the visuomotor mapping and the US is the subsequent motor action; on each trial the subject predicts the optimal motor action from the visual feedback from the previous trial. In the RW learning model equations below (eq 15 and 16) a prediction error, $\delta$, is generated on each trial from the difference between the sensory input, $u$, and the change in association between the CS and US on the previous trial, $v^{(k-1)}$, (Eq. 15). The change in association for the current trial, $v^{(k)}$, is determined by weighting the prediction error by the salience of the CS, $\alpha$, and adding this to the change in association from the previous trial (Eq. 16). This update equation modulates the association between the CS and US based on the given sensory input and a constant, individual learning rate.

$$
\begin{aligned}
& \delta^{(k)}=u^{(k)}-v^{(k-1)} \\
& v^{(k)}=v^{(k-1)}+\alpha * \delta^{(k)}
\end{aligned}
$$

Each model is characterised by its (log) model evidence (LME), which quantifies the model's goodness of fit relative to its complexity. We compared the LME across participants and these two models using the random effects BMS function implemented in SPM 12 (Stephan, Penny, Daunizeau, Moran, \& Friston, 2009).

\section{EEG Data Analysis: Pre-processing}

Data were pre-processed using SPM 12. EEG data were filtered using a highpass filter at $1 \mathrm{~Hz}$ and a low pass filter at $100 \mathrm{~Hz}$ and downsampled to $400 \mathrm{~Hz}$. Bad channels were identified using the 'threshold z-scored difference data' detection algorithm in SPM with a threshold of 8 . If $20 \%$ of the continuous data for a channel was above this threshold, it was flagged as a bad channel and removed from analysis at a later stage. Due to the length of the trials being analysed topography-based artefact correction was applied to the continuous data to remove eyeblinks. Epoched EEG data were aligned to: 1) the onset of the GO signal with a time window of $-6000 \mathrm{~ms}$ to $1000 \mathrm{~ms}$ to investigate foreperiod EEG activity, and 2) to movement offset, defined as the time the participant reached the target, with a time window of $-1000 \mathrm{~ms}$ to $2000 \mathrm{~ms}$ to investigate post-movement EEG activity. For the time-frequency analysis the power of the EEG signal at 
each frequency from 1 to $99 \mathrm{~Hz}$ was estimated using the wavelet transform in SPM. A Morlet wavelet with 7 cycles for each frequency was used.

To measure topographic changes in beta oscillatory activity over time, time frequency data was averaged over $15-30 \mathrm{~Hz}$. Data was log transformed and mean corrected such that the data at each sample point represented the change in beta power from the total average beta power over the entire epoch for each channel. Scalp-by-time analyses revealed a significant event-related decrease in beta power during motor preparation at the onset of the GO signal and also a significant event-related increase in beta power at $1000 \mathrm{~ms}$ post-movement (Figure 3A-E). A single ROI over sensorimotor cortex was generated by thresholding the group level t-statistic images at these two time points at $t=3.61$ ( $p<0.001$ uncorrected) and overlaying the two significant clusters to create one ROI that incorporated both the ERD and ERS (Figure 3F,G,H). To measure time-frequency changes over sensorimotor cortex, EEG data were averaged over the ROI electrodes selected, log transformed and mean corrected such that the data at each sample point at each frequency band represented the change in the power from the total average power for that frequency.

For 14 subjects analysed a technical error during recording meant that the rebound period for one condition (no angular perturbation, high visual noise) was 1s less than the other conditions; therefore, these trials ( $n=53$ per subject) were removed from the EEG analysis for movement offset only for these participants. However, this did not affect the main results as regression analyses were used and there was still a large number of remaining trials. It is important to note that this did not affect the pre-movement results.

\section{EEG Data Analysis: Statistical analysis}

The time-frequency data files for each trial were converted into 2D images for statistical analysis in SPM. At the single subject level, images aligned to the GO signal or movement offset were regressed against the behavioural task trajectories of either the design variables or the HGF variables using a GLM (see Figure 4 for details of the regressors used). For the design trajectories, the GLM included regressors of repetition number, noise level and visuomotor rotation for both pre-movement and post-movement EEG data. When analysing pre-movement data aligned to the GO signal, design information from the previous trial rather than the current trial was used as this would have been the only information known to the participant at the time. For the HGF trajectories, specific regressors from the first (lowest) level of the hierarchy were used to analyse the EEG data before and after a movement. For images aligned to the GO signal the following HGF trajectories were used: the prediction mean regarding the expected visuomotor rotation for the current trial $\left(\hat{x}_{1}\right)$ and prior precision $\left(\hat{\pi}_{1}\right)$. For images aligned to movement offset the following HGF trajectories were used: sensory PE $\left(\delta_{u}\right)$, precision $\left(\psi_{1}\right)$ and the posterior mean of the prediction $\left(\mathrm{x}_{1}\right)$. As there was no explicit modulation of validity at the second (highest) level of the HGF and a lack of any probabilistic structure in the trajectories of hidden states at this level, regressors from the second level were not analysed. The contrast images from all analyses for each participant were smoothed using the "SPM_smooth" function with a Gaussian kernel with a FWHM of $2 \mathrm{~Hz}$ and $150 \mathrm{~ms}$. The smoothed contrast images for each regressor of interest were then analysed at the group level using a one sample t-test to identify any EEG activity in the time-frequency domain that showed a consistent correlation 
with each regressor of interest across participants. All statistical analyses were corrected for family-wise errors within SPM using random field theory.

We hypothesised that sensorimotor beta power $(12-30 \mathrm{~Hz})$ would likely correlate with Bayesian model parameters within two windows of interest prior to and following movement based on the task design and the average change in beta power over time across participants. For images aligned to the GO signal, a window of interest was selected between the ready signal and GO signal in order to measure activity in this time period that may be relevant for motor preparation, therefore a small volume correction with a $18 \mathrm{~Hz}$ by $1000 \mathrm{~ms}$ window centered at $500 \mathrm{~ms}$ and $21 \mathrm{~Hz}$ (mid-way between $12 \mathrm{~Hz}$ and $30 \mathrm{~Hz}$ ) was used. For images aligned to movement offset the average time-frequency spectrum showed a significant increase in beta power post-movement with the peak voxel at $21 \mathrm{~Hz}$ and $1133 \mathrm{~ms}$ therefore a small volume correction in a $18 \mathrm{~Hz}$ by $2000 \mathrm{~ms}$ window centred on here was used to include the whole rebound period (see Figure 3).

\section{RESULTS}

\section{Behavioural results: Participants behaved differently under high and low visual noise}

Out of 24 participants, 19 successfully adapted their behaviour to the visuomotor rotation as shown by a significant mean decrease in initial angular error from the first APS trial to the tenth repetition of that perturbation (all $\mathrm{p}<0.05$ ). The 5 participants that did not show a significant difference in angular error by the tenth repetition were excluded from subsequent analyses for not completing the task appropriately. In the remaining sample of 19 participants, a $2 \times 2 \times 10$ repeated measures ANOVA, comparing visual noise (no or high), size of change in angular rotation on an APS trial (small, $30^{\circ}$ or large, $60^{\circ}$ ) and repetition number, was conducted for initial angular error. As expected there was a significant main effect of repetition number $\left(F(2.58,46.48)=104.15, p<0.001(G G), \eta_{p}^{2}=0.85\right.$; Figure 5A). Participants adapted quickly to the perturbation over repetitions of the same visual rotation: overall mean angular error significantly decreased from repetition 1 to repetition 3 and then remained stable for subsequent repetitions. Angular error was also modulated by the size of the perturbation as expected i.e. a large change in perturbation ( $0^{\circ}$ to $60^{\circ}$ or vice versa) generated a larger angular error than a small change in perturbation ( $0^{\circ}$ to $30^{\circ}$ or $30^{\circ}$ to $60^{\circ}$ and vice versa), but this was only significant for the APS trial and the subsequent trial (size of angular rotation $x$ repetition number: $F(4.68,84.30)=30.32, p<0.001(G G), \eta_{p}^{2}=0.63$; large change in rotation: $M \pm S D$ on APS trial $=47 \pm 6.7$; small change in rotation: $\mathrm{M} \pm \mathrm{SD}$ on APS trial $=26.9 \pm 5.1$ ).

Similar results were found for the other behavioural variables measured. Movement time was greatest on APS trials and decreased with adaptation $(F(2.02,36.39)=29.24, p<0.001(G G)$, $\eta_{\mathrm{p}}{ }^{2}=0.62$; Figure 5B). This was driven by a decrease in average velocity and an increase in path length on APS trials. Movement time was longer following a large change in perturbation compared to a small change $\left(F(1,18)=11.17, \mathrm{p}=0.004, \eta_{\mathrm{p}}{ }^{2}=0.38\right)$ and this was significantly different for the APS trial and the subsequent two trials as revealed by post-hoc t-tests over trials (angular rotation $x$ repetition number: $F(9,162)=6.58, \mathrm{p}<0.001, \eta_{\mathrm{p}}{ }^{2}=0.27$ ).

Path length was greatest on APS trials and decreased with adaptation $(F(2.37,42.73)=71.16$, $\mathrm{p}<0.001(\mathrm{GG}), \eta_{\mathrm{p}}{ }^{2}=0.80$; Figure $5 \mathrm{C}$ ). Path length was greatest following a large change in angular 
rotation compared to a small change $\left(F(1,18)=8.90, p=0.008, \eta^{2}=0.33\right)$ and this was only significant for the APS trial and repetition 3 as revealed by post-hoc t-tests (angular rotation $\mathrm{x}$ repetition number: $\left.\mathrm{F}(4.66,83.94)=16.46, \mathrm{p}<0.001(\mathrm{GG}), \eta_{\mathrm{p}}{ }^{2}=0.48\right)$. Average velocity was not significantly modulated by the size of the change in perturbation $(\mathrm{p}=0.069)$, however this was significantly modulated by repetition number $\left(\mathrm{F}(2.2,40.26)=7.57, \mathrm{p}<0.001(\mathrm{GG}), \eta_{\mathrm{p}}{ }^{2}=0.30\right)$. Participants significantly slowed down on APS trials and sped up over subsequent trials (Figure $5 \mathrm{D})$.

As expected, under high visual noise compared to no visual noise mean movement time increased (main effect of noise: $F(1,18)=64.10, p<0.001, \eta_{p}{ }^{2}=0.78$ ) and overall average velocity decreased (main effect of noise: $F(1,18)=11.75, p=0.003, \eta^{2}=0.40$ ). The rate of increase in average velocity after an APS trial was smaller under high visual noise compared to no visual noise. Participants also did not adapt as quickly or as fully under high visual noise as demonstrated by an overall increase in initial angular error (main effect of noise: $F(1,18)=11.47$, $p=0.003, \eta_{p}^{2}=0.39$ ) and path length (main effect of noise: $F(1,18)=31.81, p<0.001, \eta_{p}{ }^{2}=0.64$ ) throughout high noise blocks.

\section{Neurophysiological results: sensorimotor beta power before and after a movement modulated with behavioural adaptation}

Across participants, there was a significant positive correlation between repetition number and the PMBS such that beta power was decreased following an APS trial and increased with subsequent repetitions of the same perturbation (Figure 6A,D; peak voxel at $12 \mathrm{~Hz}, 545 \mathrm{~ms}$ : $t=7.10, p=0.001$ FWE). There was a significant effect of visual noise on beta power such that high noise blocks had higher post-movement beta power than no noise blocks; however this did not survive correction for multiple comparisons (peak voxel $23 \mathrm{~Hz}, 828 \mathrm{~ms}$ : $\mathrm{t}=3.77, \mathrm{p}=0.080$ FWE, $p=0.001$ uncorrected; Figure 6B,E). There was no significant correlation between postmovement beta power and the perturbation level (Figure 6C,F).

Task parameters from the previous trial were used to identify the neurophysiological effects of these on preparation for the next trial. We found a significant positive correlation between repetition number and beta power in the preparatory period following the ready signal and prior to the GO signal (peak voxel at $21 \mathrm{~Hz},-420 \mathrm{~ms}, \mathrm{t}=4.76, \mathrm{p}=0.018 \mathrm{FWE}$; Figure 6G,J). Beta power was significantly reduced in this preparatory period following an APS trial and increased with subsequent repetitions. There was no significant effect of visual noise and no significant effect of the perturbation level on beta power ( $p>0.1$ FWE; Figure $6 \mathrm{H}, \mathrm{I}, \mathrm{K}, \mathrm{L}$ ).

These results demonstrate that beta desynchronisation prior to movement and beta synchronisation post-movement were significantly reduced following an APS trial and increased over subsequent repetitions of the same perturbation, possibly due to diminishing PE and/or increasing precision. Thus, to formally assess the relationship between PE and precision further, we modelled the behaviour from this task using the HGF in order to produce individual trajectories of how hidden beliefs evolved throughout the task and correlated these estimated model parameters with the EEG data. 
Modelling result: the HGF readily explained participant's behaviour compared to a non-

\section{Bayesian learning model}

In order to determine that participants were behaving in a Bayes optimal manner throughout this task and therefore validate the use of the HGF in this study, we compared the ability of the Bayesian HGF and an alternative non-Bayesian learning model (the Rescorla-Wagner model, (Rescorla \& Wagner, 1972)) to explain the variance in participant's behaviour. The log model evidence (LME) from each model was compared across participants. The behavioural data used for these models was movement time as this encapsulated both the size of the angular error (due to increased path length) and any uncertainty in the movement that may be captured in participant's movement speed. Across participants, movement time was better explained using the HGF compared to the RW model (protected exceedance probability $=0.89$ i.e. the HGF was $89 \%$ more likely to explain the data better). This suggests that participants behaved in a Bayesian manner and this justifies the use of the HGF for subsequent analyses. To further test how well the HGF modelled participants behavioural data in this study, we compared the simulated movement time data from the HGF response model with the observed movement times. A linear regression analysis showed that, for all participants, observed movement times across all trials significantly predicted the simulated movement times (all $p<0.001$; group mean adjusted $\mathrm{R} 2=0.2$ ).

\section{Modelling and neurophysiological results: post-movement beta synchronisation (PMBS) correlated with parameters involved in Bayesian updating at the sensory level}

A GLM was used to identify the neurophysiological correlates of model parameters estimated from the HGF. For the post-movement period, a GLM including the following regressors was used: sensory PE $\left(\delta_{\mathrm{u}}\right)$, precision ratio $\left(\psi_{1}\right)$ and the posterior mean of the prediction $\left(\mathrm{x}_{1}\right)$. There was a significant negative correlation between the PMBS and sensory PE (peak at $15 \mathrm{~Hz}, 768 \mathrm{~ms}$; $\mathrm{t}=6.72$, $\mathrm{p}=0.001$ FWE; Figure 7A), such that the PMBS was reduced when PE was high. This mirrors the finding between the PMBS and repetition number, which acted as a proxy for adaptation. Beta power was also significantly negatively correlated with the precision ratio (peak at $16 \mathrm{~Hz}, 1788 \mathrm{~ms}$; $\mathrm{t}=5.44, \mathrm{p}=0.005 \mathrm{FWE}$; Figure $7 \mathrm{~B}$ ). When sensory precision was low, for example during blocks of high visual noise ( $\alpha$ set to high), the beta rebound was larger. In addition, the posterior mean indicating the updated belief about the size of the visuomotor rotation (which is then used as the prediction for the next trial) significantly negatively correlated with the PMBS (peak at 18Hz, 508ms; $t=4.94, \mathrm{p}=0.016 \mathrm{FWE}$; Figure 7C). The data provides evidence that the parameters involved in Bayesian updating following a movement may be encoded within the PMBS.

\section{Modelling and neurophysiological results: preparatory beta power correlated with precision, rather than error}

For the pre-movement period, a GLM using the following regressors was used to analyse preparatory beta power: predicted visuomotor rotation before any sensory input $\left(\hat{x}_{1}\right)$ and prior precision $\left(\hat{\pi}_{1}\right)$. We found a significant negative correlation between prior precision and beta power between the ready signal $(-1500 \mathrm{~ms})$ and the GO signal (0ms; peak at $14 \mathrm{~Hz},-250 \mathrm{~ms}$; $\mathrm{t}=4.66, \mathrm{p}=0.011$ FWE; Figure 8A). This shows that beta power was suppressed to a greater extent under high prior precision (least uncertainty) compared to low prior precision (highest 
uncertainty). There was no significant correlation between beta power and the estimated prediction mean $\hat{x}_{1}(p>0.05$, Figure $8 B)$.

The prior precision value in the HGF is taken from the variance of the posterior from the previous trial, which is updated based on the previous trial PE; therefore, the prior precision value incorporates the magnitude of the previous PE and an estimate of sensory uncertainty. To assess the extent to which the relation between beta power and prior precision can be explained by prior error, we conducted a series of exploratory analyses testing for the interaction between error, visual noise and beta power. For this we extracted the values of beta power in the significant cluster highlighted above (Figure 8A white dotted outline; $12-20 \mathrm{~Hz}$, $500-0 \mathrm{~ms}$ ) for trials immediately following an APS trial and APS trials +10 (behaviour fully adapted) under both high and no visual noise. A 2x2 repeated measures ANOVA revealed a significant interaction between trial number and visual noise at this time point $(F(1,18)=5.19$, $\mathrm{p}=0.035, \eta_{\mathrm{p}}{ }^{2}=0.22$ ), but no significant main effects ( $\mathrm{p}>0.1$ ). Under no visual noise there was a significant difference in the preparatory suppression of beta power following an APS trial compared to the later repetition ( $\mathrm{p}=0.036)$; beta suppression was greatest following an APS trial and increased with adaptation. There was no significant modulation of beta power with trial number for the high noise blocks $(\mathrm{p}=0.32$ ) (Figure $8 \mathrm{C}$ ). A correlation analysis of beta power over repetitions following an APS trial revealed a significant positive correlation between the beta power and repetition number for no noise blocks ( $\mathrm{r}=0.63, \mathrm{p}=0.026$; one-tailed) and no significant correlation for high noise blocks ( $\mathrm{r}=0.19, \mathrm{p}=0.31$; one-tailed) (Figure 8D). This analysis dissociates the precision-weighting from the magnitude of the previous error and therefore supports the hypothesis that preparatory beta power in the $500 \mathrm{~ms}$ prior to the GO signal readily modulates depending on movement uncertainty or prior precision rather than error.

\section{DISCUSSION}

The aim of this study was to orthogonalise the parameters of Bayesian learning in a visuomotor adaptation paradigm in order to determine their neurophysiological correlates within the sensorimotor system. We modulated visual noise independently from an angular perturbation and used the HGF to estimate trial-wise modulations of modelled parameters (prediction, PEs and precision), which explained the effect of these task inputs on participant's behaviour. In line with previous literature (H. Tan, Jenkinson, et al., 2014; H. Tan, Zavala, et al., 2014; Huiling Tan et al., 2016; Torrecillos et al., 2015), we found that the PMBS readily tracked adaptation error in the task. The PMBS also correlated with precision estimates supporting recent evidence that the PMBS does not solely modulate with PE (Huiling Tan et al., 2016), but also represents uncertainty estimates important for determining how readily the PE updates motor plans. In addition, we found that preparatory decreases in beta power were more readily modulated by uncertainty not error from the previous trial. Overall, the data suggest that sensorimotor beta power may readily reflect the precision-weighting afforded to PEs.

Previous studies have consistently found a correlation between the PMBS and PE during sensorimotor learning tasks, which is supported by the findings here. The PMBS tracked repetition number and significantly negatively correlated with sensory PE, from the first level of 
the HGF, across participants. Although there was only a trend for a negative relationship between the visual noise manipulation and the PMBS, there was a consistent negative correlation between the precision ratio and the PMBS. This demonstrates the increased sensitivity of using latent variables estimated from computational models when determining the neurophysiological correlates of behaviour (Brodersen et al., 2013) and supports theories that the PMBS does not solely modulate with PE.

Previous studies have highlighted that the PMBS readily represents changes in model uncertainty associated with our prior beliefs about the sensory consequences of our movements. However, here we manipulated sensory uncertainty and saw similar modulations in the PMBS. This discrepancy can be resolved by understanding computationally how modulating different forms of uncertainty, or precision, modulate the precision-weighting of PE. Indeed, in the HGF, there is a precision ratio consisting of sensory precision from the level below (numerator) and prior precision from the level being updated (denominator), which denotes the weighting given to the PE and dictates how readily the model will be updated. Therefore, manipulating sensory and prior precision has opposing effects on this precisionweighting. Tan et al (2016) found that increasing model uncertainty correlated with a decrease in the average power of the PMBS. According to the HGF, decreasing prior precision (inverse model uncertainty) in this way would increase the precision-weighting of PEs creating a negative relationship between precision-weighting and the PMBS. Indeed, in the current study, we found this same negative relationship, however by modulating sensory uncertainty. This suggests that independent of whether model uncertainty or sensory uncertainty is modulated, the PMBS negatively correlates with the resultant precision-weighting afforded to PEs. Sensorimotor beta oscillations may therefore represent the summation of these different uncertainty values, which are potentially encoded by different inputs into the sensorimotor cortex.

Moreover, in our study, pre-movement preparatory beta power between the ready signal and the GO signal correlated with precision in the prediction of the sensory consequences of movement, but not with the estimated prediction mean. Following the ready signal there was a decrease in beta power in preparation to move and this decrease was greater for trials with higher prior precision (less model uncertainty). This is in line with previous findings that beta power during motor preparation was dependent on directional uncertainty such that beta power decreased more when there was less uncertainty in the target location (Tzagarakis et al., 2015). This suggests that similar associations with beta power and uncertainty can be seen irrespective of the exact nature of the uncertainty. The prior precision parameter produced by the HGF is the predicted model precision before any sensory input has been received and comes from the posterior precision from the previous trial. This regressor therefore readily modulated with the precision-weighting of the previous PE, which was heavily influenced by the external changes in sensory uncertainty, therefore does not purely represent prior precision without any influence of sensory uncertainty. Indeed, this regressor modulated very closely with the block-wise changes in visual noise. The data here are supported by a recent paper showing that foreperiod beta power is not related to primary motor output, but more likely reflects higher order processing of sensory afference needed for successful sensorimotor adaptation (Alayrangues et al., 2019). 
To probe the relationship between error, uncertainty and preparatory beta power further, we carried out additional exploratory analyses in this time window by comparing beta power on trials with high and low error and high and low visual noise in a $2 \times 2$ factorial design. This aimed to orthogonalise the effects of error and noise on beta power. We found a significant interaction between these factors before the GO signal to move. Beta power was suppressed significantly more under no visual noise than high visual noise on the trial immediately following an APS trial despite the same initial angular error being produced in the previous trial; however, this difference disappeared with adaptation. This suggests that preparatory beta power was not specifically modulated by sensory uncertainty or error. Pre-movement beta power reflected the relative contribution of sensory and model uncertainty estimates as represented in the precision ratio. When there is no visual uncertainty, a new perturbation immediately increases the weighting on sensory information such that the precision-weighted PE more readily updates the model and adapts to the new context. This is associated with a large decrease in beta power, which then increases with adaptation. However, under high visual noise, sensory precision is suppressed such that model precision remains high despite the introduction of an angular perturbation and does not change as readily over subsequent trials due to the decreased precision-weighting of PEs. Beta power remains high over subsequent trials despite the magnitude of the PE changing with adaptation. This suggests that preparatory beta power more readily tracks this precision-weighting (in an inverse relationship) rather than the magnitude of the PE. Therefore, this study shows that beta power both prior to and following a movement is negatively associated with the precision-weighting afforded to sensory prediction errors from the previous or the current trial respectively.

In support of this hypothesis, a recent study by (Vilares \& Kording, 2017) showed that PD patients, who have increased resting beta power and reduced sensorimotor beta modulation with movement (S. Little, Pogosyan, Kuhn, \& Brown, 2012), placed less weight on sensory evidence needed to make Bayes optimal decisions in a visual discrimination task. This can be likened to a reduced precision-weighting of sensory PEs. Moreover, dopaminergic medication (which decreases beta power) has been shown to modulate responses to low-level sensory PEs of stimulus outcomes (Bestmann, Ruge, Rothwell, \& Galea, 2014; Iglesias et al., 2013). As well as guiding decisions, it has been suggested that modulations in sensory precision may be causally involved in motor initiation based on active inference (Palmer et al, 2016; Friston et al, 2015). Indeed, the akinetic role of beta power in PD and healthy controls and the association between beta power and sensory precision in this study supports these ideas.

It is important to understand the neurophysiological basis of beta oscillations in order to determine how this activity may influence behaviour. Precision is thought to be encoded by synaptic gain (post-synaptic responsiveness) of superficial pyramidal cells in the cortex (K. Friston, 2005; K. Friston \& Kiebel, 2009); therefore, the neuromodulation of synaptic gain (precision) by dopamine (known to modulate beta power) provides a potential mechanism by which changes in precision can modulate beta power. Alternatively, fast, synchronous oscillatory activity can itself modulate synaptic gain of coupled neurons (Chawla, Lumer, \& Friston, 1999; K. J. Friston, Bastos, Pinotsis, \& Litvak, 2015). This suggests that oscillatory activity could have a mechanistic impact on neuronal processing and may not simply be an epiphenomenon of population activity. The timing of neuromodulatory changes in the brain can be quite slow, therefore it is likely that rapid attentional mechanisms that appear to modulate responses under different levels of uncertainty most likely rely on an interaction between 
neuromodulatory and electrophysiological mechanisms, which remains to be elucidated. However, based on this evidence it is feasible that beta oscillatory activity measured with EEG may correlate with changes in precision parameters (synaptic gain).

The results described here should be contrasted with the predictive coding literature in which beta oscillations are thought to transmit descending predictions rather than encoding ascending precision-weighted PEs within a canonical microcircuit (Arnal \& Giraud, 2012; Arnal et al., 2011; Bastos et al., 2012). Evidence for an attention-dependent gain control by high frequency gamma rather than beta oscillatory activity mainly comes from electrophysiological data from the auditory and visual cortices, therefore the sensorimotor system may not adhere to this scheme (Adams, Shipp, \& Friston, 2013). Indeed, the different cytoarchitecture of the motor cortex and the complex interaction with spinal cord circuitry may alter the oscillatory dynamics within this system. More work is needed to understand how oscillatory activity within different systems is generated in order to identify potentially differing roles of the same frequency of oscillatory activity in different brain regions.

This study demonstrates that sensorimotor beta oscillations may play an important role in Bayesian updating and the representation of uncertainty during visuomotor adaptation. Specifically, we found that sensorimotor beta oscillations may mediate the precision-weighting of PEs, which is represented following a movement during Bayesian updating and in the precision surrounding the predictions of a movement before the next trial. Importantly, although activity at the macro-scale correlates with estimated model parameters, this does not provide evidence that the brain computes and uses these latent variables. Cross-modal research integrating single cell, local field potential and EEG recordings during behaviour is essential to fully determine how this oscillatory activity is processed by the brain and used to guide behaviour. 


\section{REFERENCES}

Adams, R. A., Shipp, S., \& Friston, K. J. (2013). Predictions not commands: active inference in the motor system. Brain Structure and Function, 218(3), 611-643. https://doi.org/10.1007/s00429-012-0475-5

Alayrangues, J., Torrecillos, F., Jahani, A., \& Malfait, N. (2019). Error-related modulations of the sensorimotor post-movement and foreperiod beta-band activities arise from distinct neural substrates and do not reflect efferent signal processing. NeuroImage, 184, 10-24. https://doi.org/10.1016/j.neuroimage.2018.09.013

Arnal, L. H., \& Giraud, A.-L. (2012). Cortical oscillations and sensory predictions. Trends in Cognitive Sciences, 16(7), 390-398. https://doi.org/10.1016/j.tics.2012.05.003

Arnal, L. H., Wyart, V., \& Giraud, A.-L. (2011). Transitions in neural oscillations reflect prediction errors generated in audiovisual speech. Nature Neuroscience, 14(6), 797-801. https://doi.org/10.1038/nn.2810

Bastian, A. J. (2006). Learning to predict the future: the cerebellum adapts feedforward movement control. Current Opinion in Neurobiology, 16(6), 645-649. https://doi.org/10.1016/j.conb.2006.08.016

Bastos, A. M., Usrey, W. M., Adams, R. A., Mangun, G. R., Fries, P., \& Friston, K. J. (2012). Canonical Microcircuits for Predictive Coding. Neuron, 76(4), 695-711. https://doi.org/10.1016/j.neuron.2012.10.038

Bays, P. M., \& Wolpert, D. M. (2007). Computational principles of sensorimotor control that minimize uncertainty and variability. The Journal of Physiology, 578(2), 387-396.

Bestmann, S., Ruge, D., Rothwell, J., \& Galea, J. M. (2014). The Role of Dopamine in Motor Flexibility. Journal of Cognitive Neuroscience, 27(2), 365-376. https://doi.org/10.1162/jocn_a_00706

Blakemore, S.-J., \& Sirigu, A. (2003). Action prediction in the cerebellum and in the parietal lobe. Experimental Brain Research, 153(2), 239-245. https://doi.org/10.1007/s00221-003$1597-\mathrm{z}$ 
Brodersen, K. H., Deserno, L., Schlagenhauf, F., Lin, Z., Penny, W. D., Buhmann, J. M., \& Stephan, K. E. (2013). Dissecting psychiatric spectrum disorders by generative embedding. NeuroImage : Clinical, 4, 98-111. https://doi.org/10.1016/j.nicl.2013.11.002

Chawla, D., Lumer, E. D., \& Friston, K. J. (1999). The relationship between synchronization among neuronal populations and their mean activity levels. Neural Computation, 11(6), 1389-1411.

Cohen, L. G., \& Starr, A. (1987). Localization, timing and specificity of gating of somatosensory evoked potentials during active movement in man. Brain: A Journal of Neurology, 110 ( Pt 2), 451-467.

Davis, N. J., Tomlinson, S. P., \& Morgan, H. M. (2012). The Role of Beta-Frequency Neural Oscillations in Motor Control. Journal of Neuroscience, 32(2), 403-404. https://doi.org/10.1523/JNEUROSCI.5106-11.2012

Engel, A. K., \& Fries, P. (2010). Beta-band oscillations--signalling the status quo? Current Opinion in Neurobiology, 20(2), 156-165. https://doi.org/10.1016/j.conb.2010.02.015

Friston, K. (2005). A theory of cortical responses. Philosophical Transactions of the Royal Society of London. Series B, Biological Sciences, 360(1456), 815-836.

https://doi.org/10.1098/rstb.2005.1622

Friston, K. J., Bastos, A. M., Pinotsis, D., \& Litvak, V. (2015). LFP and oscillations-what do they tell us? Current Opinion in Neurobiology, 31, 1-6. https://doi.org/10.1016/j.conb.2014.05.004

Friston, K., \& Kiebel, S. (2009). Predictive coding under the free-energy principle. Philosophical Transactions of the Royal Society B: Biological Sciences, 364(1521), 1211-1221. https://doi.org/10.1098/rstb.2008.0300

Friston, K., Mattout, J., \& Kilner, J. (2011). Action understanding and active inference. Biological Cybernetics, 104(1-2), 137-160. https://doi.org/10.1007/s00422-011-0424-z

Iglesias, S., Mathys, C., Brodersen, K. H., Kasper, L., Piccirelli, M., den Ouden, H. E. M., \& Stephan, K. E. (2013). Hierarchical prediction errors in midbrain and basal forebrain during 
sensory learning. Neuron, 80(2), 519-530.

https://doi.org/10.1016/j.neuron.2013.09.009

Körding, K. P., \& Wolpert, D. M. (2004). Bayesian integration in sensorimotor learning. Nature, 427(6971), 244-247. https://doi.org/10.1038/nature02169

Little, S., Pogosyan, A., Kuhn, A. A., \& Brown, P. (2012). $\beta$ band stability over time correlates with Parkinsonian rigidity and bradykinesia. Experimental Neurology, 236(2), 383-388. https://doi.org/10.1016/j.expneurol.2012.04.024

Little, Simon, \& Brown, P. (2014). The functional role of beta oscillations in Parkinson's disease. Parkinsonism and Related Disorders, 20, S44-S48. https://doi.org/10.1016/S13538020(13)70013-0

Mathys, C. D., Lomakina, E. I., Daunizeau, J., Iglesias, S., Brodersen, K. H., Friston, K. J., \& Stephan, K. E. (2014). Uncertainty in perception and the Hierarchical Gaussian Filter. Frontiers in Human Neuroscience, 8. https://doi.org/10.3389/fnhum.2014.00825

Mathys, C., Daunizeau, J., Friston, K. J., \& Stephan, K. E. (2011). A bayesian foundation for individual learning under uncertainty. Frontiers in Human Neuroscience, 5, 39. https://doi.org/10.3389/fnhum.2011.00039

Palmer, C., Zapparoli, L., \& Kilner, J. M. (2016). A New Framework to Explain Sensorimotor Beta Oscillations. Trends in Cognitive Sciences, $0(0)$. https://doi.org/10.1016/j.tics.2016.03.007

Rescorla, R., \& Wagner, A. (1972). A theory of pavlovian conditioning: variations in the effectiveness of reinforcement and nonreinforcement, in Clsssical Conditioning II: Current Research and Theory (pp. 64-99). New York: Appleton Century Crofts.

Starr, A., \& Cohen, L. G. (1985). “Gating” of somatosensory evoked potentials begins before the onset of voluntary movement in man. Brain Research, 348(1), 183-186. https://doi.org/10.1016/0006-8993(85)90377-4 
Stephan, K. E., Penny, W. D., Daunizeau, J., Moran, R. J., \& Friston, K. J. (2009). Bayesian model selection for group studies. NeuroImage, 46(4), 1004-1017. https://doi.org/10.1016/j.neuroimage.2009.03.025

Tan, H., Jenkinson, N., \& Brown, P. (2014). Dynamic Neural Correlates of Motor Error Monitoring and Adaptation during Trial-to-Trial Learning. Journal of Neuroscience, 34(16), 56785688. https://doi.org/10.1523/JNEUROSCI.4739-13.2014

Tan, H., Zavala, B., Pogosyan, A., Ashkan, K., Zrinzo, L., Foltynie, T., ... Brown, P. (2014). Human subthalamic nucleus in movement error detection and its evaluation during visuomotor adaptation. The Journal of Neuroscience: The Official Journal of the Society for Neuroscience, 34(50), 16744-16754. https://doi.org/10.1523/JNEUROSCI.341414.2014

Tan, Huiling, Wade, C., \& Brown, P. (2016). Post-Movement Beta Activity in Sensorimotor Cortex Indexes Confidence in the Estimations from Internal Models. The Journal of Neuroscience: The Official Journal of the Society for Neuroscience, 36(5), 1516-1528. https://doi.org/10.1523/JNEUROSCI.3204-15.2016

Torrecillos, F., Alayrangues, J., Kilavik, B. E., \& Malfait, N. (2015). Distinct Modulations in Sensorimotor Postmovement and Foreperiod $\beta$-Band Activities Related to Error Salience Processing and Sensorimotor Adaptation. The Journal of Neuroscience, 35(37), 1275312765. https://doi.org/10.1523/JNEUROSCI.1090-15.2015

Tzagarakis, C., West, S., \& Pellizzer, G. (2015). Brain oscillatory activity during motor preparation: effect of directional uncertainty on beta, but not alpha, frequency band. Frontiers in Neuroscience, 9. https://doi.org/10.3389/fnins.2015.00246

Vilares, I., \& Kording, K. P. (2017). Dopaminergic medication increases reliance on current information in Parkinson's disease. Nature Human Behaviour, 1, s41562-017-0129-017. https://doi.org/10.1038/s41562-017-0129 
Vossel, S., Mathys, C., Daunizeau, J., Bauer, M., Driver, J., Friston, K. J., \& Stephan, K. E. (2014). Spatial Attention, Precision, and Bayesian Inference: A Study of Saccadic Response Speed. Cerebral Cortex, 24(6), 1436-1450. https://doi.org/10.1093/cercor/bhs418

Wolpert, D. M., Ghahramani, Z., \& Jordan, M. I. (1995). An internal model for sensorimotor integration. Science (New York, N.Y.), 269(5232), 1880-1882.

Wolpert, D. M., \& Miall, R. C. (1996). Forward Models for Physiological Motor Control. Neural Networks: The Official Journal of the International Neural Network Society, 9(8), 12651279. 


\section{FIGURES}

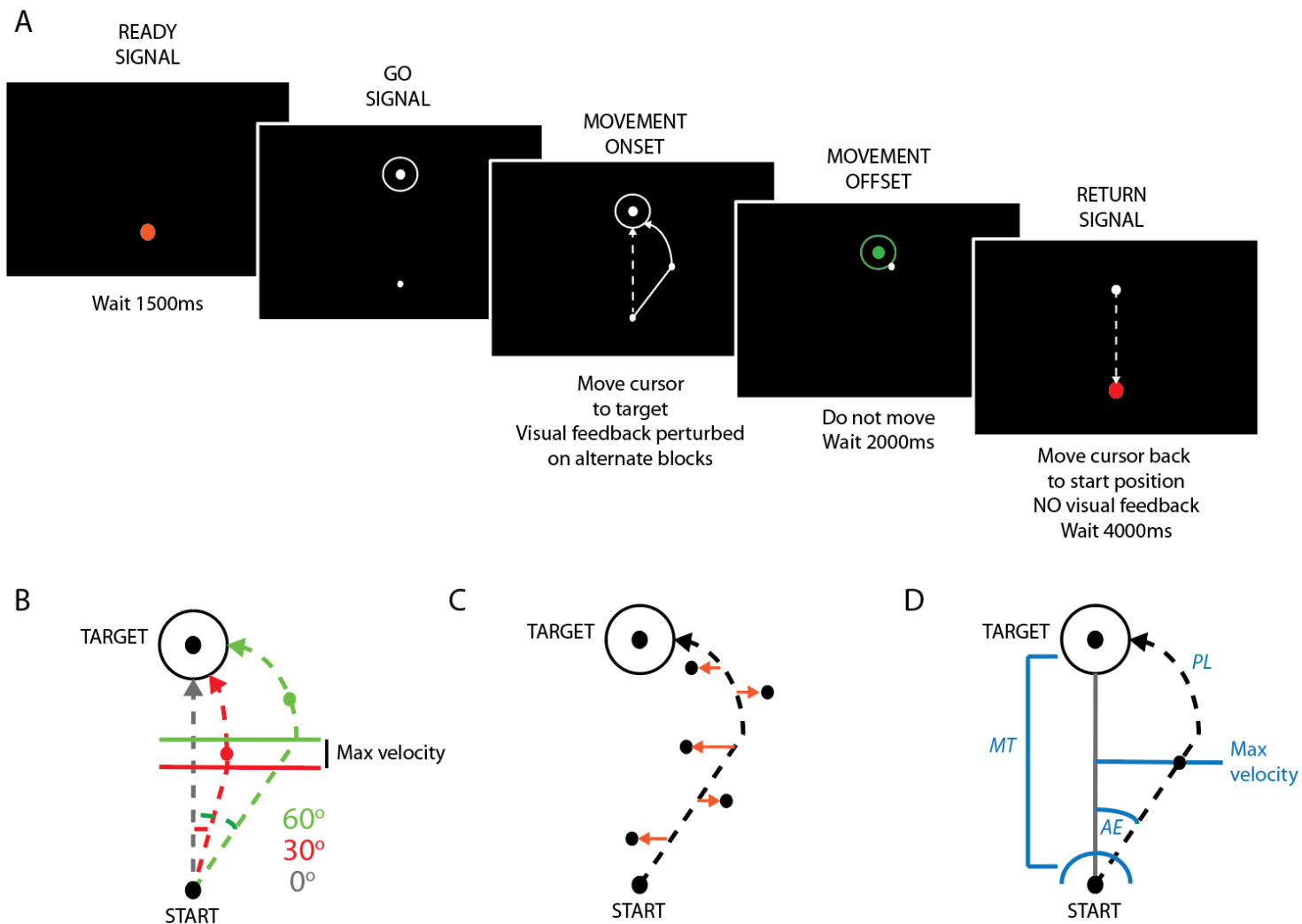

Figure 1. Visuomotor adaptation task design. A) Participants were instructed to move the cursor from the start position to the target via the most direct path using a touchpad. A visuomotor rotation was added to the feedback of the cursor. Participants were told to wait at the target until the return signal when participants were asked to move back to the start position, which was signalled with a tactile marker: no visual feedback was given.

Participants then waited for $4 s$ until the ready signal was given then another $1.5 s$ until the go signal was given for the next trial. B) Angular perturbations introduced into the visual feedback of the cursor were either $60^{\circ}, 30^{\circ}$ or veridical with the participant's finger movement. The same perturbation remained constant for up to 15 trials and participants were required to adapt to the angular perturbation over trials in order to find the most direct path to the target. C) Sensory uncertainty was modulated by adding noise into the visual feedback on alternate blocks. On noise trials the cursor was randomly shifted along the X-axis continually throughout the movement such that the cursor appeared to jitter. This made the true movement trajectory uncertain. D) A number of behavioural dependent variables were calculated from the movement trajectories on each trial. Angular error (AE) was calculated as the angle between a line connecting the start position and the target and a line connecting the start position and the point of maximum velocity. Movement initiation was calculated as the point at which the cursor crossed a semi-circular boundary with a radius of $5 \mathrm{~mm}$ from the start position. Movement time (MT) was calculated as the length of time from movement initiation until movement offset when the cursor reached the outer ring of the target zone. Path length (PL) was calculated as the sum of the differentiated cursor position from the time of movement initiation to the time of movement offset. 


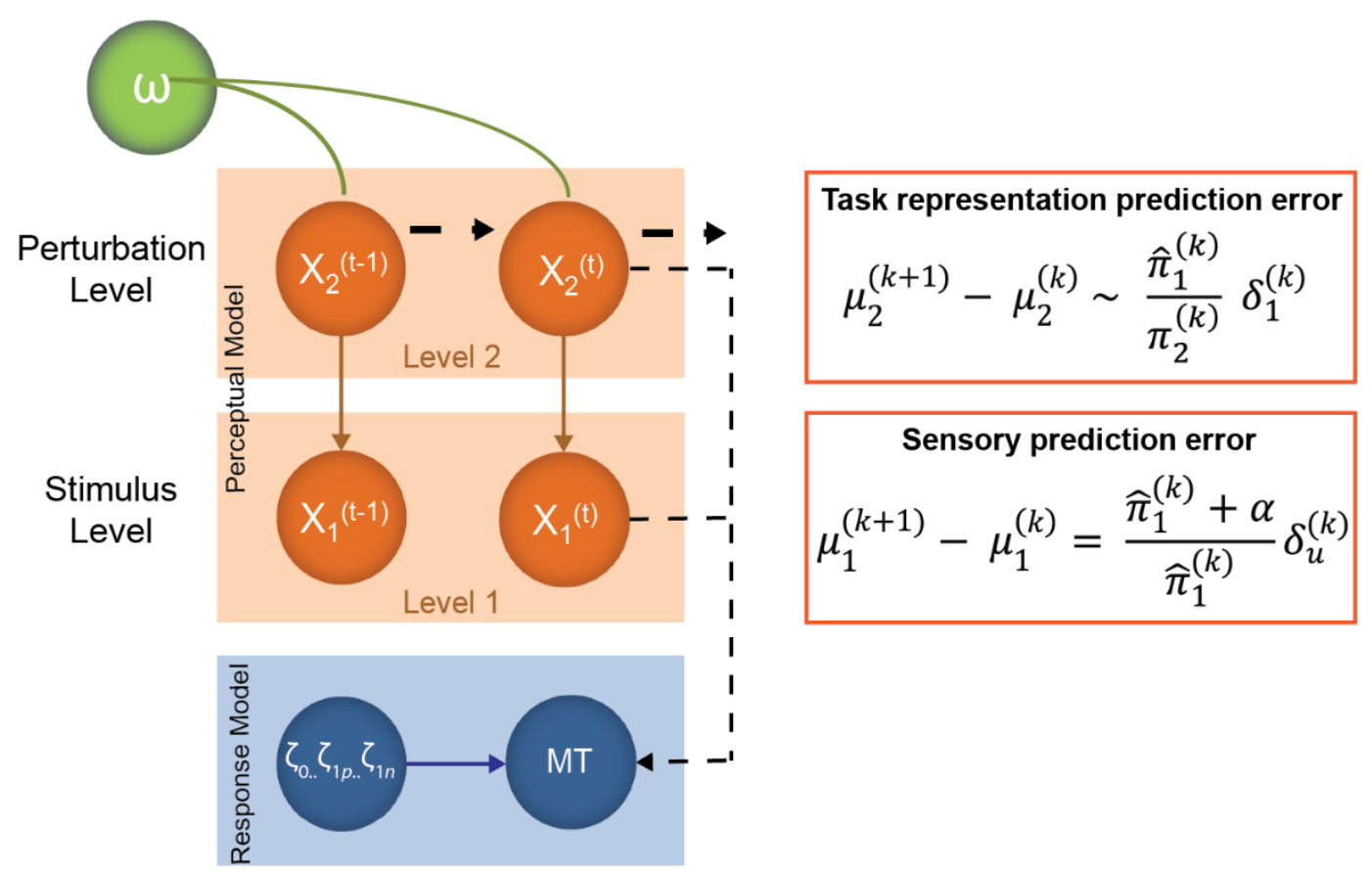

Figure 2. Overview of the HGF used in this study. In this study a 2 level HGF was employed. At the first level, the perceptual model estimates how participant's beliefs about the perturbation level $x 1$ modulate trial-by-trial i.e. what perturbation level governs that trial. This prediction depends on the current task representation level and the magnitude of noise in the visual feedback $\alpha$ (assumed to be constant for a given block). Sensory PEs at this level represent the error between this prediction and the actual perturbation level that is weighted by the sensory uncertainty. At the second level, the perceptual model estimated the probability with which the perturbation level would change over trials, $x 2$. This level describes the validity of the perturbation level and the variance of this depends on the individualised learning step size $\omega$. As there was no explicit modulation of validity at this level we only analysed the data from the first level. The response model maps these hidden beliefs onto observed movement times in order to estimate individual parameters that dictate learning rate based on Vossel et al (2014). MT = movement time. 

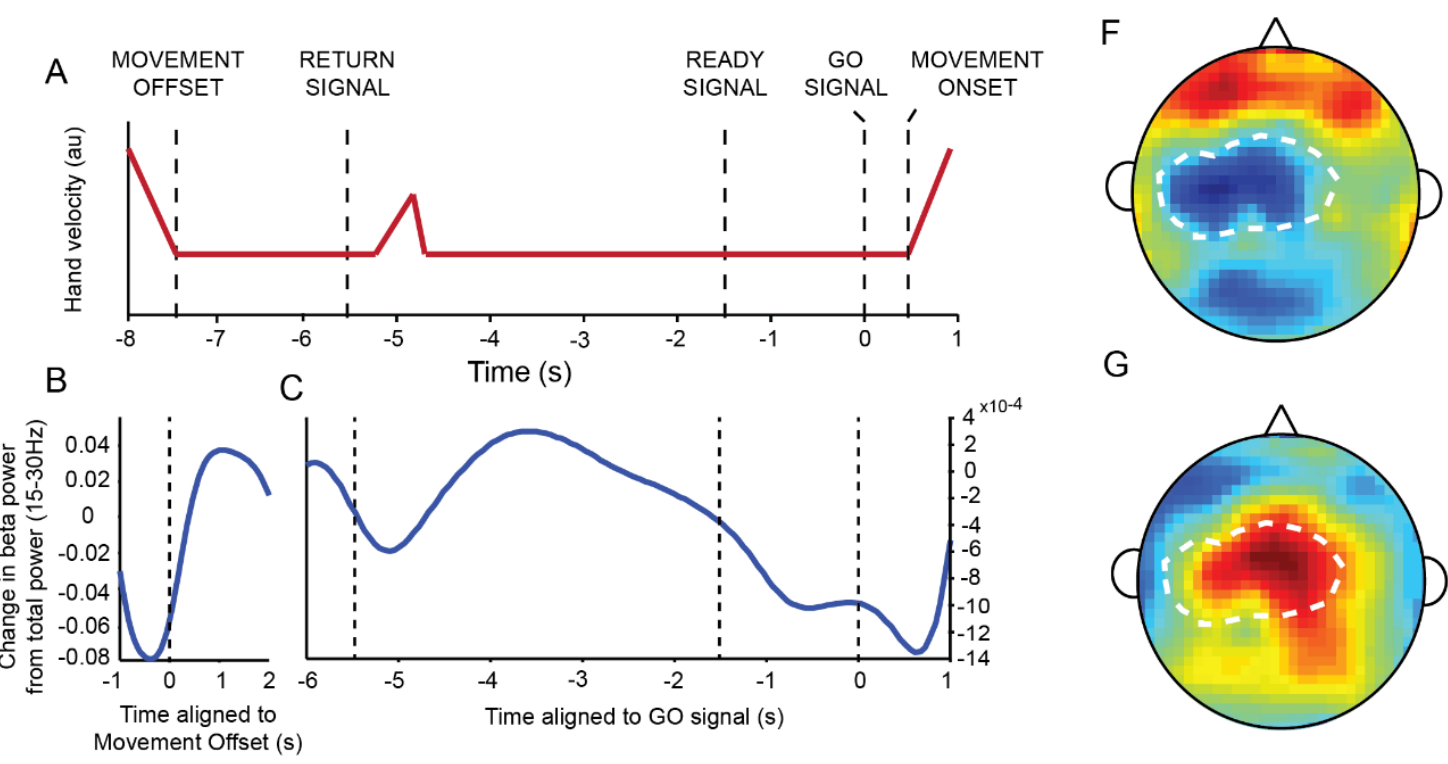

G

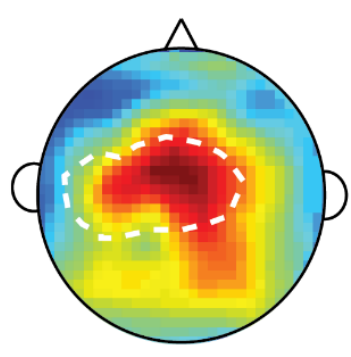

D

E
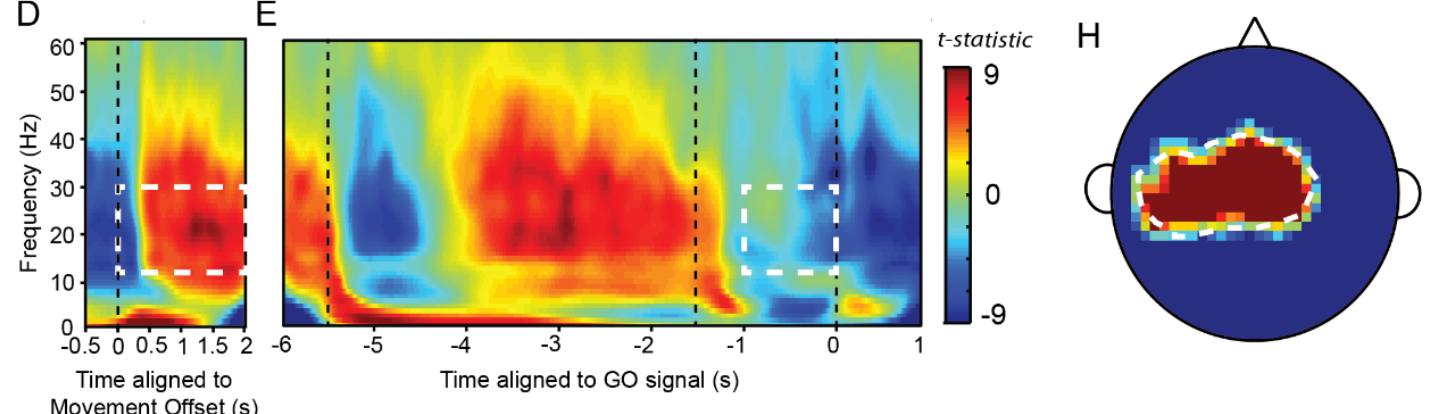

Figure 3. Beta power (15-30Hz) modulated over sensorimotor cortex with movement. A) A schematic demonstrating the change in hand velocity throughout the task with each signal. This highlights the points at which participants were moving and the points they remained still. $B+C)$ EEG data averaged over beta power (15$30 \mathrm{~Hz}$ ) over a selected ROI and over subjects in an epoch aligned to movement offset (B) and the GO signal (C). $D+E)$ Time-frequency plots demonstrating the average change in power over time across $1 \mathrm{~Hz}$ frequency bands from 1-60Hz averaged over subjects. EEG data were epoched around movement offset (D) and the GO signal (E). F-H) An ROI was selected over sensorimotor cortex. The white dotted line represents the ROI selected as shown in $H$. This incorporated both the average ERD over subjects at the onset of the GO signal (F) and the average ERS over subjects $1 \mathrm{~s}$ after movement offset $(G)$. The white dotted squares indicate the windows of interest that were used for small volume correction for statistical analyses. 


\section{A DESIGN REGRESSORS}

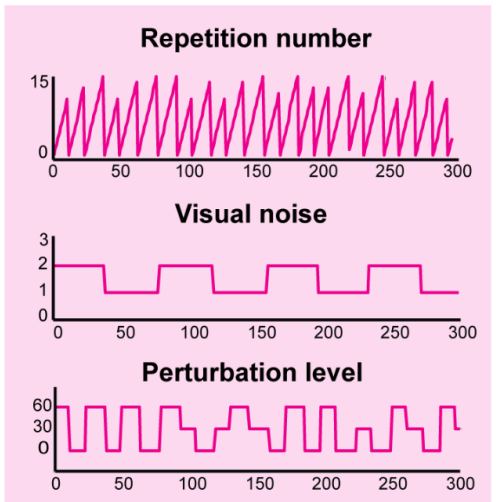

C BEHAVIOUR

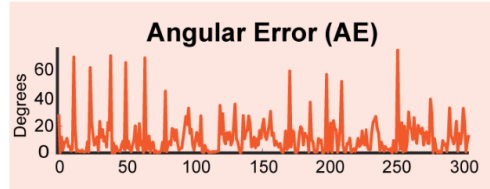

Movement Time (MT)

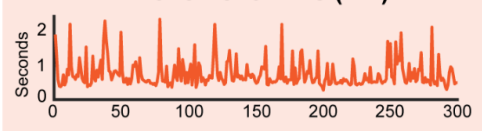

B HGF REGRESSORS
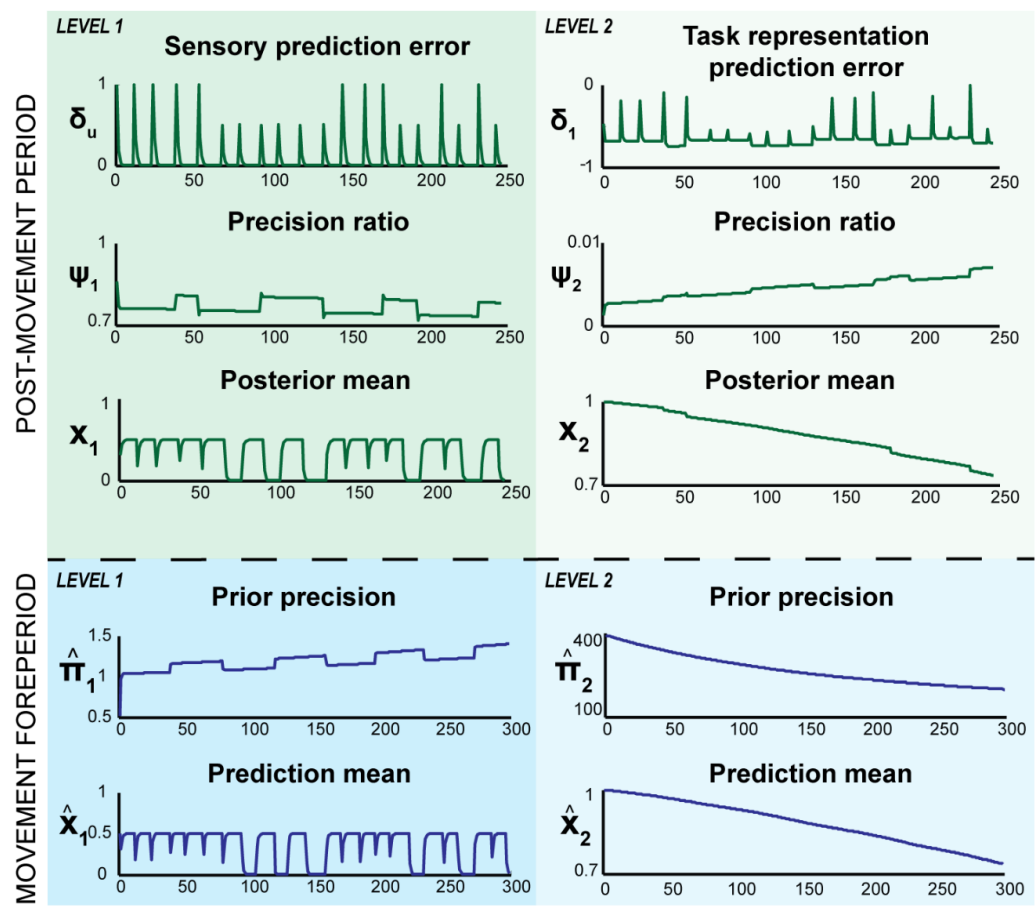

Figure 4. The trial-wise trajectories of task inputs or hidden beliefs estimated by the HGF. A) The task was designed to manipulate perturbation level and visual noise orthogonally. Repetition number follows the implicit changes in perturbation level. Repetition 1 is the first introduction of a new perturbation: the perturbation switch (PS) trial. Visual noise was modulated in blocks of high and low visual noise. Angular rotation denotes the size of the perturbation level introduced $\left(0^{\circ}, 30^{\circ}\right.$ or $\left.60^{\circ}\right)$, which stayed constant over subsequent repetitions of that perturbation. These regressors were used in a GLM to explain sensorimotor beta power following a movement and before a movement. B) Estimated trajectories from the HGF demonstrating how different hidden beliefs evolved over time throughout the task are shown for an example participant. Hidden beliefs estimated after sensory input was received were used to explain the post-movement beta synchronisation (upper panel) and predictions made before sensory input was received were used to explain preparatory beta power before movement onset (lower panel). These trajectories were produced at the first level of the HGF. C) Raw behavioural data from an exemplar subject showing angular error and movement times across all trials. Movement time was the observed behaviour input into the HGF to model hidden beliefs across trials. 

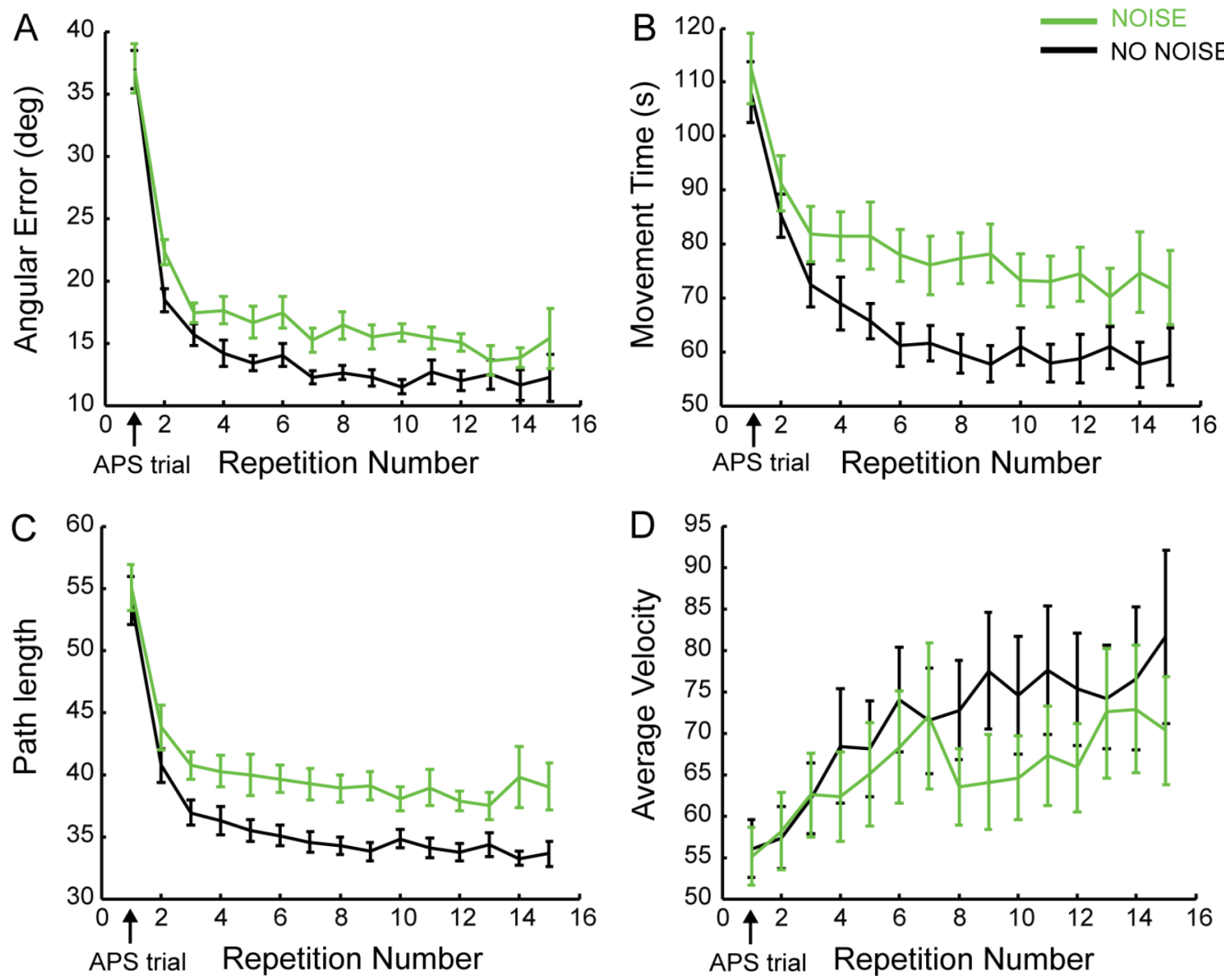

Figure 5. The visuomotor rotation and visual noise significantly modulated behaviour. All behavioural variables were averaged over all perturbation sizes for the noise (green) and no noise (black) blocks and for each repetition of a perturbation. A) Angular error was maximal on an angular perturbation switch (APS) trial and decreased over subsequent repetitions with adaptation. B) Movement time was on average greater for high visual noise compared to no visual noise conditions. C) Path length modulated in a very similar way to angular error decreasing with repetition number. On average, path length was significantly greater for high visual noise compared to no visual noise conditions. D) Average velocity decreased on APS trials and increased over subsequent repetitions with adaptation. Average velocity was significantly less under high visual noise compared to no visual noise. Error bars = standard error of the mean (sem). 


\section{POST-MOVEMENT PERIOD}

A Repetition number
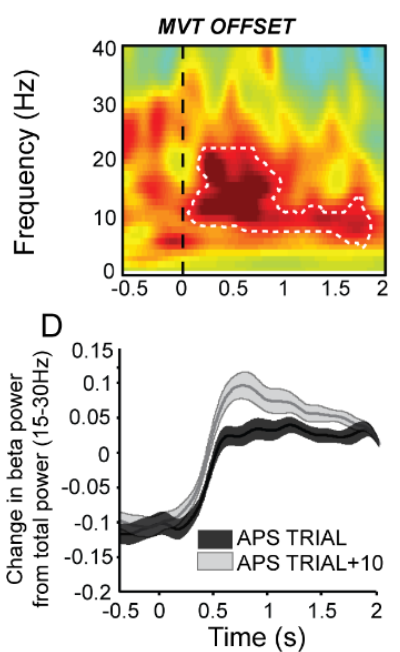

MOVEMENT FOREPERIOD
B Visual noise

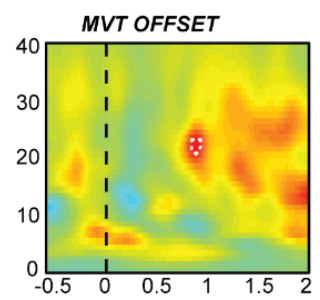

$\mathrm{E}$

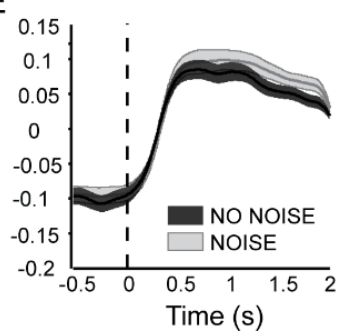

C Angular rotation

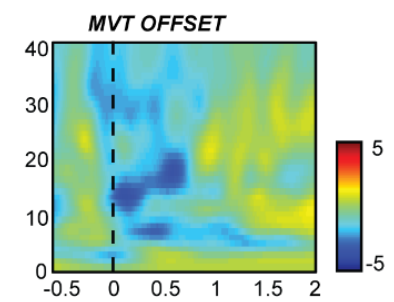

$\mathrm{F}$

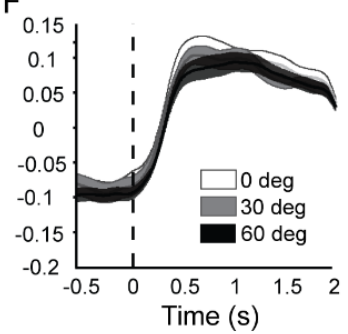

G Repetition number

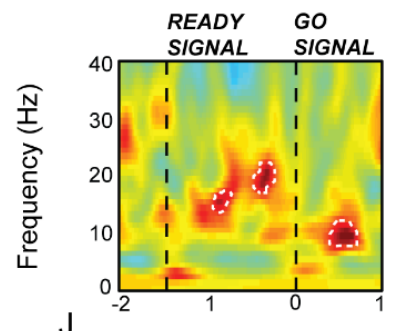

$\checkmark$

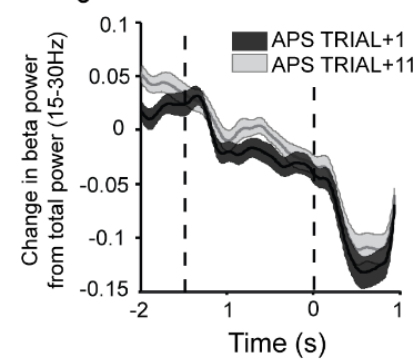

$\mathrm{H}$

H Visual noise
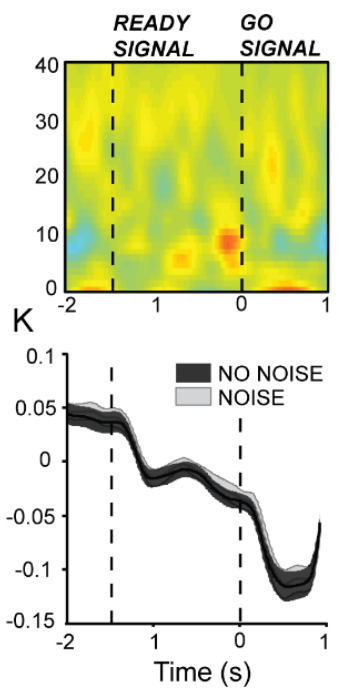

I Angular rotation

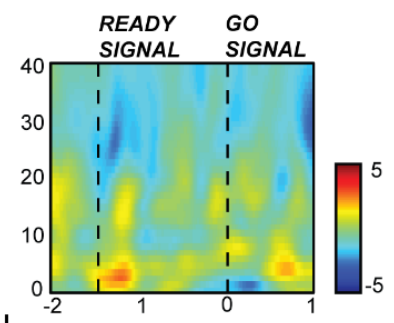

$\mathrm{L}$

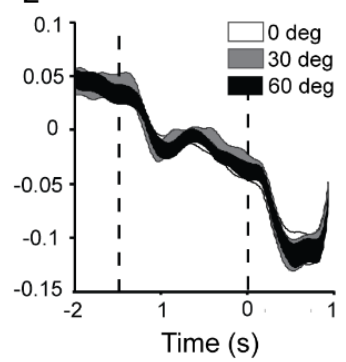

Figure 6. Beta power before and after a movement correlated with repetition number. $A-C, G-I) A G L M$ involving the design regressors (repetition number, visual noise and angular rotation) measured the correlation between EEG activity and each regressor of interest for each subject individually for the time period after each movement $(A-C)$ and before each movement $(G-I)$. Here the time-frequency plots show the $t$-statistic from a series of one sample t-tests measuring the consistency of these relationships across participants. The results show areas in which the data was consistently positively correlated (warm colours) or negatively correlated (cool colours) with the regressor of interest across participants. $D-F, J-L)$ These graphs display beta power (13-30Hz) averaged across participants over trials in a particular condition. D+J) Beta power averaged over angular perturbation switch (APS) trials (or APS trials +1 for pre-movement period) and adapted trials (APS trial +10 orA PS trials +11 for pre-movement period) to compare activity on trials with a large error and no error. E+K) Beta power averaged over high visual noise and low visual noise blocks. $F+L$ ) Beta power averaged over all trials with a particular sized angular rotation: 0 degrees, 30 degrees or 60 degrees. The upper two rows show data corresponding to the post-movement period and the bottom two rows correspond to the pre-movement period. White dotted lines show significant activity thresholded at $t=3.61, p<0.001$ uncorrected. Beta power following a movement positively correlated with repetition number consistently across subjects (peak voxel at $12 \mathrm{~Hz}, 545 \mathrm{~ms}$ : $t=7.10, p=0.001 \mathrm{FEW} ; A, D$ ) and visual noise (peak voxel $23 \mathrm{~Hz}, 828 \mathrm{~ms}: t=3.77, p=0.080 \mathrm{FWE}, p=0.001$ uncorrected; $B, E)$. Beta power in the preparatory period following the ready signal and prior to the GO signal significantly correlated with repetition number (peak voxel at $21 \mathrm{~Hz},-420 \mathrm{~ms}, t=4.76, p=0.018 \mathrm{FWE}$; $\mathrm{G}$,J). Beta power was significantly reduced in this preparatory period following an APS trial and increased with subsequent repetitions. Shaded error bands $=$ sem. 


\section{POST-MOVEMENT PERIOD}

A Prediction error

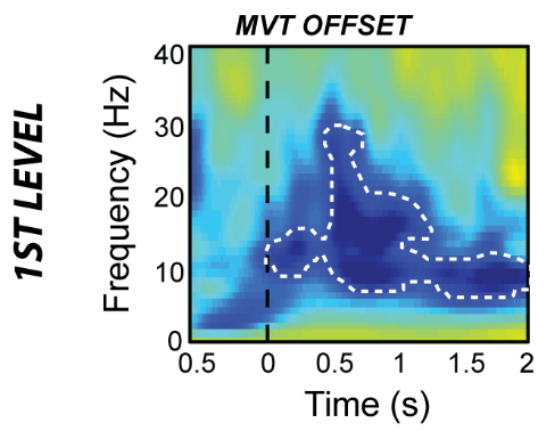

B Precision ratio

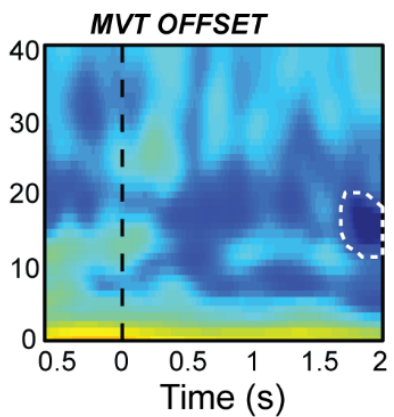

C Posterior mean

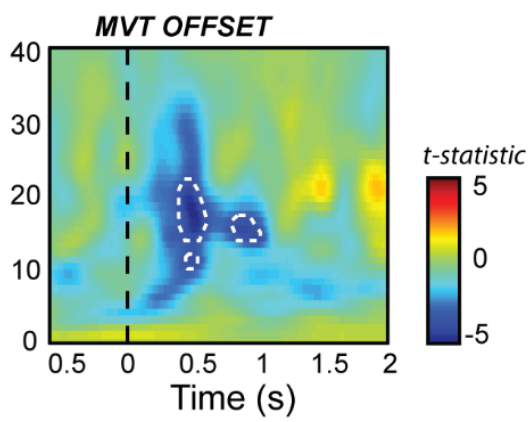

Figure 7. Post-movement beta synchronisation correlates with multiple components of Bayesian updating. A GLM involving regressors estimated from the first level of the HGF measured the correlation between EEG activity following a movement and each regressor of interest for each subject individually. Here the timefrequency plots show the $t$-statistic from a series of one sample t-tests measuring the consistency of these relationships across participants. There was a significant consistent negative correlation between the PMBS and prediction error (A), the precision ratio (B) and the posterior mean $(C)$ estimated at the first level of the HGF across participants. White dotted lines show significant activity thresholded at $t=3.61, p<0.001$ uncorrected. 


\section{MOVEMENT FOREPERIOD}
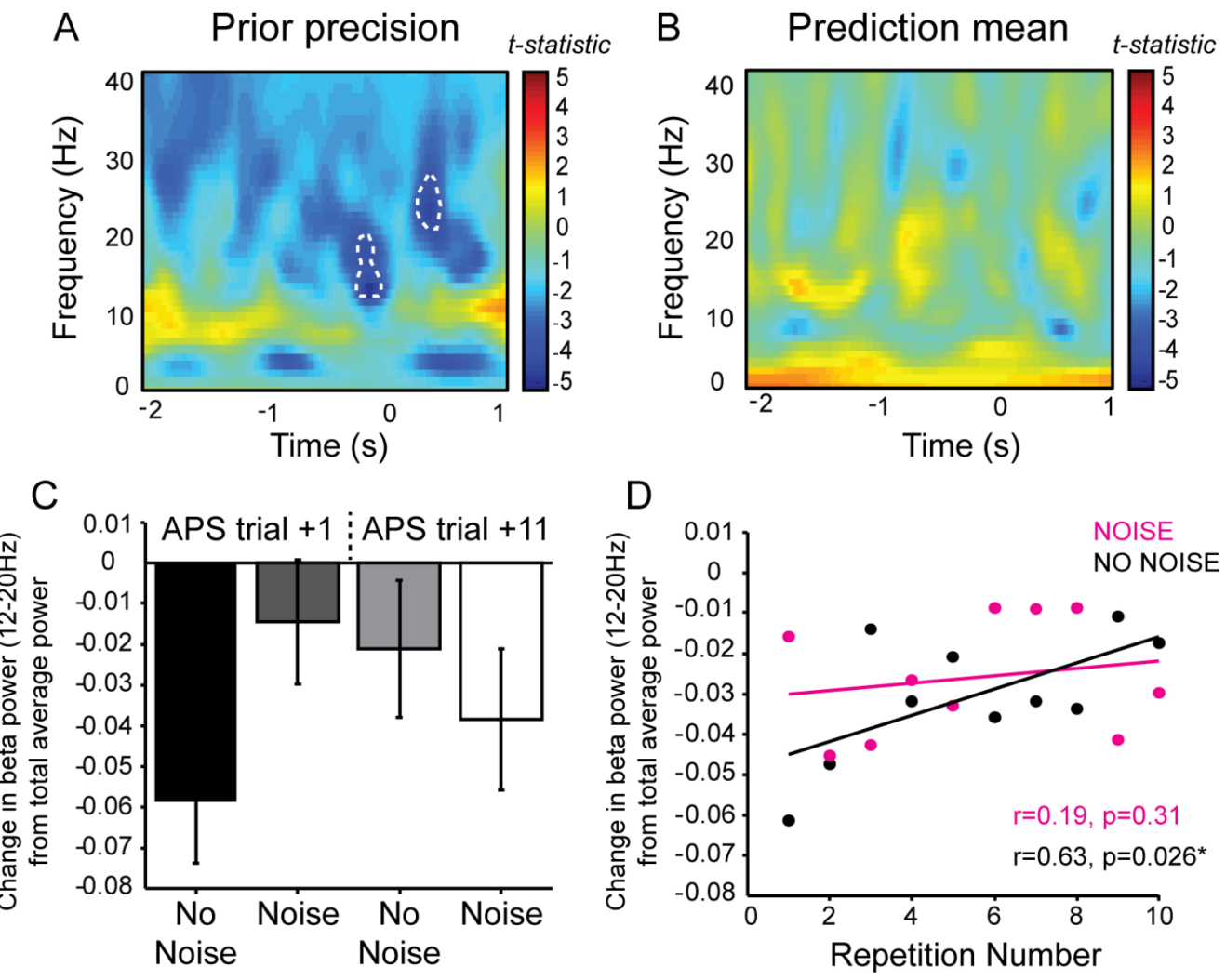

Figure 8. Preparatory beta power inversely correlated with prior precision. $A+B)$ Time-frequency plots showing the results of a one sample t-test measuring the consistency of the correlation between the estimated parameters of the HGF and the EEG data. Low-frequency beta power (12-20Hz) was consistently negatively correlated across participants with prior precision (A). There were no significant consistent correlations for the prediction mean (B). C) Beta power was averaged over $12-20 \mathrm{~Hz}$ and over trials for four conditions in a $2 \times 2$ factorial design (visual noise level (high vs low); repetition number (angular perturbation switch (APS) trial $+1 \mathrm{vs}$ $P S$ trial +11$)$. There was a significant interaction between visual noise and repetition number $(p=0.035$,

Eta2 $=0.22)$. D) There was a significant correlation between repetition number and beta power $(12-20 \mathrm{~Hz})$ for the no noise condition ( $r=0.63, p=0.026$; one-tailed): beta power increased with adaptation over repetitions of the same perturbation. There was no significant correlation between repetition and beta power for the high noise condition ( $r=0.19, p=0.31$; one-tailed). White dotted lines show significant activity thresholded at $t=3.61, p<0.001$ uncorrected. Error bars $=$ sem. 